\title{
Performance of CS Fallback for Long Term Evolution Mobile Network
}

\author{
Ren-Huang Liou, Yi-Bing Lin, Fellow, IEEE, Yingrong Coral Sung, Pang-Cheng Liu, and \\ Christian Wietfeld, Senior Member, IEEE
}

\begin{abstract}
When a mobile operator migrates its network from the third generation (3G) system to Long Term Evolution (LTE), both $3 G$ and LTE will coexist for a period of time. Since the 3G circuit-switched (CS) voice mechanism is more mature and available than that for the LTE Voice over Internet Protocol (VoIP), the operator may consider CS fallback (CSFB) as a solution for providing reliable voice calls. According to the 3G Partnership Project (3GPP) CSFB procedure, when a mobile user in the LTE network has an incoming or an outgoing call, the user equipment (UE) falls back from LTE to Universal Mobile Telecommunications System (UMTS). When the call is complete and released, the UE immediately returns to LTE. If the next activity for the UE is another voice call, immediately switching from UMTS to LTE may not be efficient. In this case, the UE has to perform another CSFB. To resolve this issue, we suggest delaying the returns to avoid unnecessary CSFBs, which is called delayed return (DR). Based on the measurements from the real UMTS and LTE networks, we develop an analytic model for investigating the performance of the CSFB with DR. This paper indicates that the DR scheme can effectively reduce the CSFB costs by up to $60 \%$.
\end{abstract}

Index Terms-Circuit-switched fallback (CSFB), delayed return (DR) scheme, Long Term Evolution (LTE).

Manuscript received October 16, 2013; revised December 4, 2013; accepted January 18, 2014. Date of publication January 27, 2014; date of current version October 14, 2014. The work of R.-H. Liou was supported by the MediaTek Fellowship. The work of Y.-B. Lin was supported in part by the National Science Council of Taiwan under Grant NSC 102-2221-E-009-056 and Grant NSC 103-2218-E-009-010, by Academia Sinica under Grant AS102-TP-A06, by Chunghwa Telecom, by the National Chiao Tung University and Industrial Technology Research Institute (ITRI) Joint Research Center Project, by the Information and Communications Research Laboratories/ITRI Project, by the Department of Industrial Technology Academic Technology Development Program under Grant 102-EC-17-A-03-S1-193, and by the Aiming for the Top University and Elite Research Center Development Plan of the Ministry of Education of Taiwan. The work of Y. C. Sung was supported by Chunghwa Telecom. The work of C. Wietfeld was supported in part by Deutsche Forschungsgemeinschaft under the Collaborative Research Center SFB 876 "Providing Information by Resource-Constrained Analysis" project B4. The review of this paper was coordinated by Prof. N. Kato.

R.-H. Liou and P.-C. Liu are with the Department of Computer Science, National Chiao Tung University, Hsinchu 300, Taiwan (e-mail: rhliou@ @ cs.nctu. edu.tw; pcliu@cs.nctu.edu.tw).

Y.-B. Lin is with the Department of Computer Science, National Chiao Tung University, Hsinchu 300, Taiwan, and also with the Institute of Information Science and the Research Center for Information Technology Innovation, Academia Sinica, Taipei 115, Taiwan (e-mail: liny@cs.nctu.edu.tw).

Y. C. Sung is with the Center for Information and Communications Technology, National Chiao Tung University, Hsinchu 300, Taiwan (e-mail: ycsung@ cs.nctu.edu.tw).

C. Wietfeld is with the Communications Networks Institute, Technische Universität Dortmund, Dortmund 44227, Germany (e-mail: christian.wietfeld@ tu-dortmund.de).

Color versions of one or more of the figures in this paper are available online at http://ieeexplore.ieee.org.

Digital Object Identifier 10.1109/TVT.2014.2302832

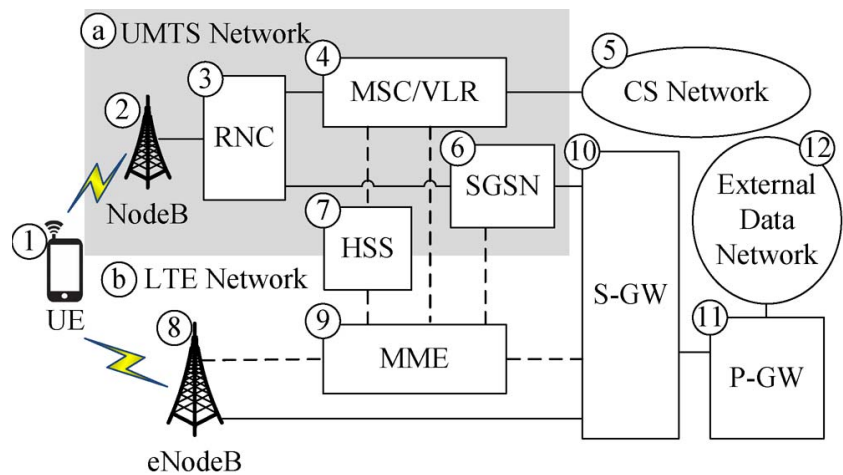

Fig. 1. EPS and UMTS architecture for CSFB (dashed lines: signaling; solid lines: signaling/data).

\section{INTRODUCTION}

$\mathbf{T}$ HE Third Generation Partnership Project (3GPP) Long Term Evolution (LTE) [1] defines an all IP network architecture that evolves from the Universal Mobile Telecommunications System (UMTS) [2] to provide high-speed data services. When a mobile operator migrates its network from the $3 \mathrm{G}$ system to LTE, both $3 \mathrm{G}$ and LTE will coexist for a period of time, and initially, the $3 \mathrm{G}$ coverage is more complete than the LTE coverage. Since LTE is a packet-switched (PS) network, the circuit-switched (CS) voice service [3] is not as supported as the 3G. Therefore, the LTE voice service is offered through the Voice over Internet Protocol (VoIP) technology. Since the 3G CS voice mechanism is more mature and available than that for LTE VoIP, in the deployment of LTE, many operators consider CS fallback (CSFB) [4] as the solution for providing reliable voice calls. The CSFB technique switches the user equipment (UE) or the mobile phone from the LTE network to the $3 \mathrm{G}$ legacy system when a voice call is attempted. To use the CS domain in the UMTS system when the UE resides in LTE, the LTE network needs to register the UE with both the LTE and the UMTS CS domain and delivers the CS paging message from UMTS to the UE.

Fig. 1 shows a simplified architecture of UMTS and evolved packet system (EPS) for LTE. This architecture includes two parts: the UMTS network and the LTE network. A UE [see (1) in Fig. 1] accesses UMTS and LTE services through the radio interfaces. In the UMTS network [see (a) in Fig. 1], the UMTS Terrestrial Radio Access Network (UTRAN) consists of NodeBs [see (2) in Fig. 1] and radio network controllers (RNCs) [see (3) in Fig. 1]. A NodeB provides wideband codedivision multiple-access radio connectivity between the UE and 
the corresponding RNC. The RNC connects to the UMTS core network. This core network is partitioned into the CS and PS domains. The CS domain includes mobile switching centers (MSCs) and visitor location registers (VLRs) [see (4) in Fig. 1]. An MSC is responsible for call control and connection between the UE and the external CS Network [see (5) in Fig. 1]. A VLR is responsible for the mobility activities of the MSC. The PS domain consists of serving GPRS support nodes (SGSNs) [see (6) in Fig. 1], which provide the mobility and session services to the mobile phones. The home subscriber server (HSS) [see (7) in Fig. 1] is the master database containing all user-related subscription information, which supports mobility management of mobile users. In the LTE network [see (b) in Fig. 1], the Evolved UMTS Terrestrial Radio Access Network (E-UTRAN) consists of evolved NodeBs (eNodeBs) [see (8) in Fig. 1] to offer LTE radio connectivity to the UE. The E-UTRAN connects to the LTE core network that includes the following components. A mobility management entity (MME) [see (9) in Fig. 1] interacts with the HSS to offer mobility management and session control. The serving gateway (S-GW) [see (10) in Fig. 1] is responsible for routing data packets and is an anchor of the user plane data for intrasystem and intersystem handovers. The packet data network gateway (P-GW) [see (11) in Fig. 1] provides the connectivity to the external data network [see (12) in Fig. 1] and the per-user based packet filtering. In the UMTS PS domain, the SGSNs connect to the external data network through the S-GW and the P-GW. According to the 3GPP CSFB procedure [4], when a mobile user in the LTE network has an incoming or an outgoing call, the UE falls back from LTE to UMTS. When the call is complete and released, the UE immediately returns to LTE. If the next activity for the UE is another voice call, immediately switching from UMTS to LTE may not be efficient. In this case, the UE has to perform another CSFB. To resolve this issue, we suggest delaying the returns to avoid unnecessary CSFBs.

This paper is organized as follows. Section II describes the CSFB procedures, the existing immediate return (IR) scheme, and the proposed delayed return (DR) scheme for returning to LTE. Section III proposes an analytic model for the IR and DR schemes. Section IV studies the performance of IR and DR by numerical examples, and conclusions are given in Section V.

\section{Third Generation Partnership Project Circuit-Switched Fallback Procedures}

Here, the CSFB procedures defined in the 3GPP, including call setup and call release with IR, are described. We also report the measured processing times for the procedures collected in live $3 \mathrm{G}$ and LTE networks in [5]-[7]. Then, we introduce the DR scheme, including call release and data session setup.

\section{A. LTE Call Setup With CS Fallback}

Fig. 2 shows the CSFB message flow when a UE makes a call in the LTE network. The following steps are executed.

Step 1: The UE sends the Extended Service Request message to the MME to initiate the CSFB procedure.

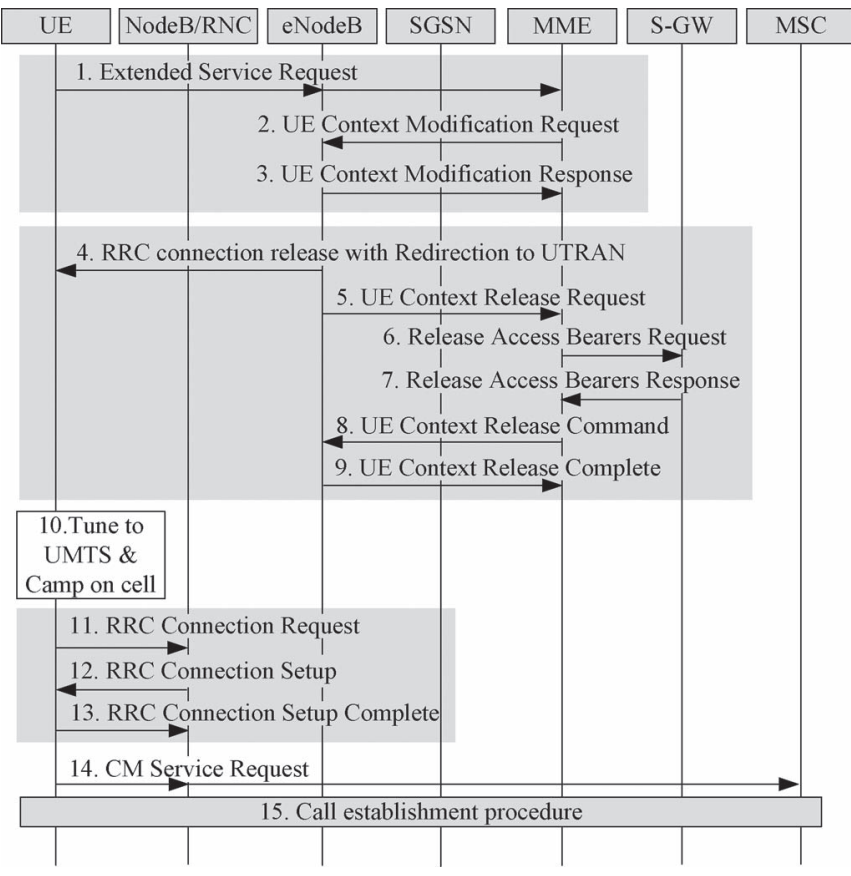

Fig. 2. Call setup with CSFB.

Steps 2 and 3: The MME exchanges the UE Context Modification Request and Response message pair with the eNodeB to indicate that the UE should fall back to the UTRAN. Note that Steps 1-3 take about $0.3 \mathrm{~s}$ [5].

Step 4: The eNodeB sends the UE the radio resource control (RRC) Connection Release with Redirection to UTRAN message to indicate that it may follow the cell identity and system information to attach to the corresponding UTRAN cell.

Steps 5-9: Parallel to Step 4, the eNodeB sends the UE Context Release Request message to the MME to release the bearers between the eNodeB and the S-GW. Steps 4-9 take about $0.2 \mathrm{~s}$ [5].

Step 10: After Step 4, the UE tunes the radio to UMTS and camps on the NodeB according to the system information in the RRC Connection Release with Redirection to UTRAN message. Step 10 takes about $2.3 \mathrm{~s}$ for 3 GPP R8 and $0.3 \mathrm{~s}$ for 3GPP R9 [5].

Steps 11-13: The UE exchanges with the NodeB the RRC Connection Request and Setup message pair to establish the radio connection. Then, the UE sends the NodeB the RRC Connection Setup Complete message to acknowledge the RRC connection establishment. Steps 11-13 take about $0.3 \mathrm{~s}$ [5].

Steps 14 and 15: The UE sends the call management (CM) Service Request message to initiate the CS call establishment procedure. The UE includes the CS mobile originated (CSMO) flag to indicate that it is a CSFB call. The CS call establishment at Step 15 follows the 3GPP standard (see [8] for details). Steps 14 and 15 take about $3.5 \mathrm{~s}$ [5].

Note that if the UE in LTE is engaged in a data session when a call arrives, then the PS connection (for the data session) is also switched to UMTS in the call setup of the CSFB procedure. (For the details of the PS connection switching, see [1]). 


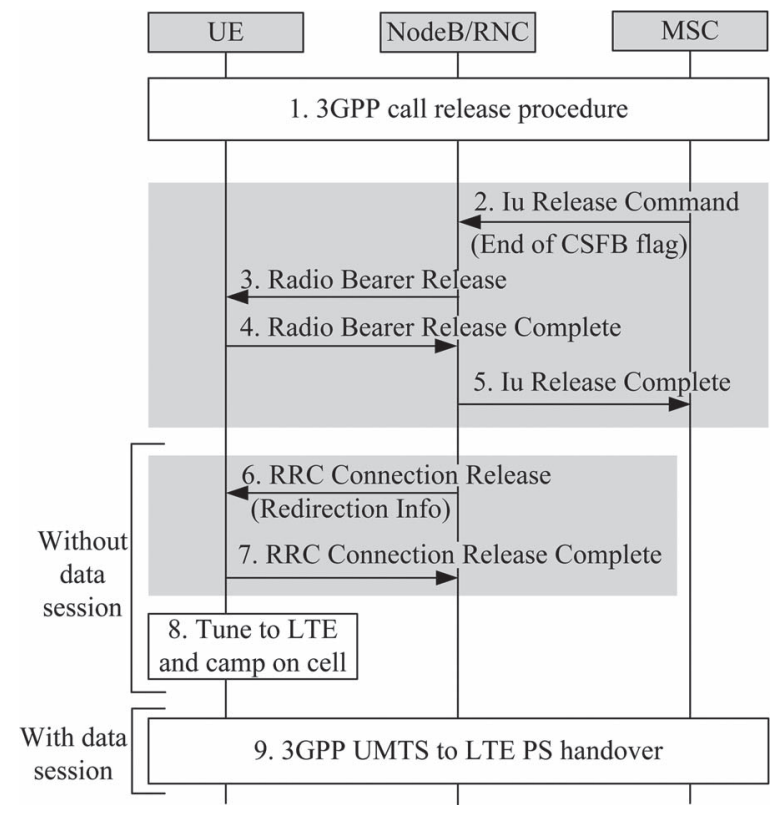

Fig. 3. Call release with IR.

\section{B. Call Release With IR}

Fig. 3 illustrates the call release procedure with IR. After a voice call is released, if no UMTS data session is in progress, the UTRAN moves the UE to the LTE network immediately with the following steps.

Step 1: The standard 3GPP call release procedure is executed [8]. Steps 2-5: The MSC sends the UTRAN the lu Release Command message to release the bearer between the MSC and the RNC. This message contains the End of CSFB flag to indicate that the call that was released is a CSFB call. Then, the NodeB sends the UE the Radio Bearer Release message to release the radio bearer between the NodeB and the UE.

Steps 6-8: According to the End of CSFB flag, the NodeB knows that the UE is LTE capable. The NodeB sends the UE the RRC Connection Release with Redirection Info message to release the radio connection between the NodeB and the UE. Then, the UE switches to the LTE network according the redirection information in the message.

If the UE is engaged in a data session when the voice call is released, then Steps 6-8 are replaced by the standard 3GPP UMTS to LTE PS handover (Step 9) [1], and the data session is moved to the LTE network.

\section{Call Release With DR}

When a voice call is released, if the UE is engaged in a data session, then it is switched back to LTE, as shown in Fig. 3 (Steps 1-5 and 9). If the UE is not engaged in a data session, then it does not need to return to LTE immediately. Fig. 4 shows the call release procedure with DR. The UE releases the radio connection and stays in UMTS in the idle mode.

Steps $1-5$ of the message flow in Fig. 4 is the same as the call release procedure with IR. At Step 6, the NodeB sends the RRC Connection Release without Redirection Info message.

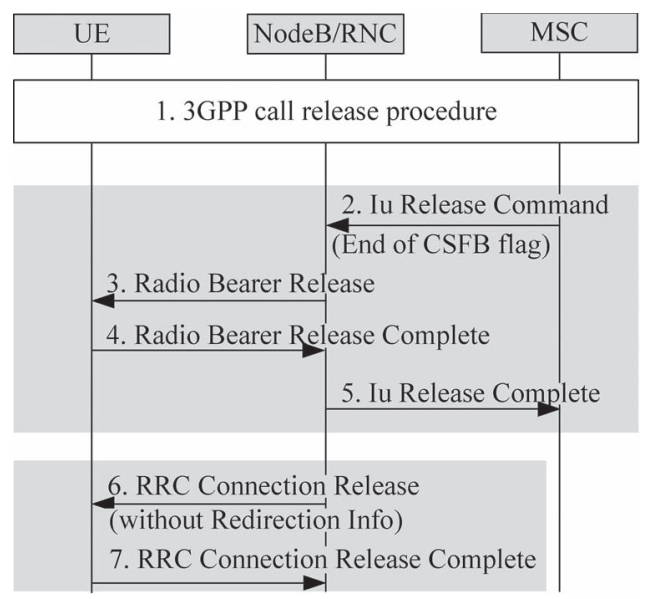

Fig. 4. Call release with DR.

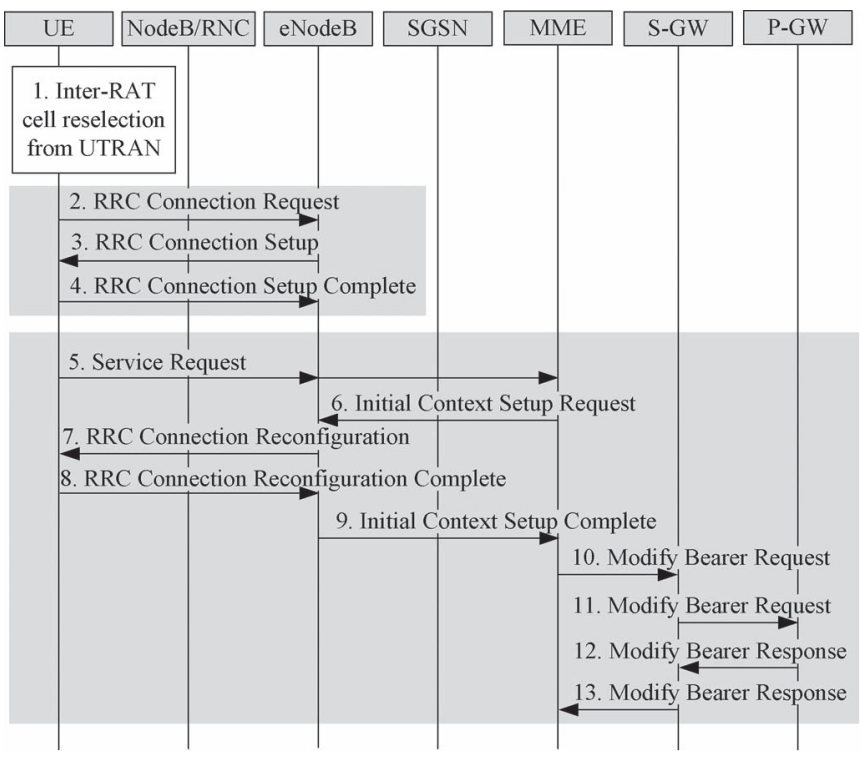

Fig. 5. Date session setup in UMTS with DR.

Because this message does not contain the optional redirection information, the UE will not switch to LTE. This message instructs the UE to release the RRC connection, to stay in UMTS, and to change its status to the idle mode. Compared with the CSFB with IR, Steps 8 and 9 in Fig. 3 are saved in the CSFB with DR.

\section{Data Session Setup in UMTS With DR}

Suppose that DR is applied, and the UE does not return to LTE after a voice call (i.e., there is no data session in progress when the voice call is released). If the next event to the UE is a data session arrival, then it will receive the PS paging message from the UMTS NodeB. The UE is switched to LTE to establish the PS connection. The detailed steps are described as follows (see Fig. 5):

Step 1: The UE executes the 3GPP Interradio access technology cell reselection procedure from UTRAN [9] to perform the measurement process and then selects an LTE cell. 
Steps 2-4: The UE exchanges with the eNodeB the RRC Connection Request and Setup message pair to establish the radio connection. Then, the UE sends the eNodeB the RRC Connection Setup Complete message to acknowledge the RRC connection establishment procedure.

Step 5: The UE sends the MME the Service Request message to initial the establishment of the bearer for the PS connection.

Steps 6-9: The MME exchanges with the eNodeB the Initial Context Setup Request and Complete messages to establish the radio bearer between the UE and the eNodeB. The request message also contains the bearer information between the eNodeB and the S-GW. The eNodeB sends the UE the RRC Connection Reconfiguration message to modify the bearer information of the radio connection. Then, the UE sends the eNodeB the RRC Connection Reconfiguration Complete message to acknowledge the radio bearer reconfiguration.

Steps 10-13: The MME sends the S-GW the Modify Bearer Request message to establish the bearer between the eNodeB and the S-GW and the bearer between the S-GW and P-GW.

We note that, for the data session setup, the CSFB with DR does not incur extra overhead over IR from the network viewpoint. Specifically, Steps 10-13 in Fig. 2 are executed by IR, which are the same as Steps 1-4 in Fig. 5. IR also executes Steps 5-13 in Fig. 5 when a data session arrives.

\section{Analytic Model}

Here, an analytic model to study the performance improvement of the DR scheme over the IR scheme is proposed. Specifically, we derive the probability $p$ that, when a voice call arrives, the UE can be connected at UMTS without CSFB due to DR. Fig. 6 shows a timing diagram for voice call arrivals (at $t_{2}$ and $t_{5}$ ) and data session arrivals (at $t_{1}$ and $t_{6}$ ). Let $t_{c}=t_{4}-t_{2}$ (also $t_{7}-t_{5}$ ) be a voice call holding time. Let the intercall arrival time $t_{a}=t_{5}-t_{4}$ be a random variable with the density function $f_{a}(\cdot)$, the distribution function $F_{a}(\cdot)$, the variance $V_{a}$, and the Laplace transform $f_{a}^{*}(s)$. Let the session holding time $t_{s}=t_{3}-t_{1}$ (also $t_{8}-t_{6}$ ) be a random variable with the mean $1 / \mu$, and the intersession arrival time $t_{p}=t_{6}-t_{3}$ be a random variable with the density function $f_{p}(\cdot)$, the variance $V_{p}$, and the Laplace transform $f_{p}^{*}(s)$. Suppose that the call release event at $t_{4}$ is a random observer of the period $\left[t_{3}, t_{6}\right]$. From the residual life theorem [10], the interval $\tau_{p}=t_{6}-t_{4}$ is the residual life of $t_{p}$ with the density function $r_{p}(\cdot)$, the distribution function $R_{p}(\cdot)$, and the Laplace transform $r_{p}^{*}(s)$. We define an observation interval as a period between when the previous call arrives and when the next call arrives (e.g., the interval $\left[t_{2}, t_{5}\right]$ in Fig. 6). It is clear that the probability $p$ described earlier is the probability that no data session is in progress when the previous call is released (with probability $p_{1}$ ) and that no data session arrives before the next voice call arrives (with probability $p_{2}$ ).

The sequence of $t_{s}$ and $t_{p}$ forms an alternating renewal process [11]; therefore, $p_{1}=\left(E\left[t_{p}\right]\right) /\left(E\left[t_{p}\right]+E\left[t_{s}\right]\right)$. Since a call release event is a random observer of $t_{s}$ and $t_{p}, p_{2}$ can be

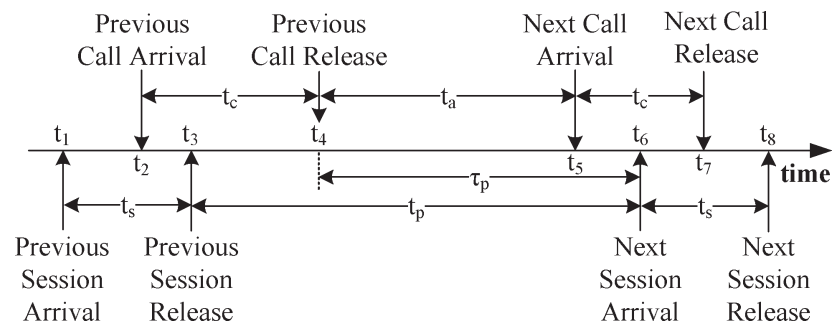

Fig. 6. Timing diagram for voice call and data session arrivals.

expressed as $\operatorname{Pr}\left[t_{a}<\tau_{p}\right]$. According to the given description, we have

$$
p=p_{1} p_{2}=\left(\frac{E\left[t_{p}\right]}{E\left[t_{p}\right]+E\left[t_{s}\right]}\right) \operatorname{Pr}\left[t_{a}<\tau_{p}\right] .
$$

Based on the inverse Laplace transform formula and the residue theorem [12], [13], $\operatorname{Pr}\left[t_{a}<\tau_{p}\right]$ in (1) is derived as

$$
\begin{aligned}
\operatorname{Pr}\left[t_{a}<\tau_{p}\right] & =\int_{\tau_{p}=0}^{\infty} r_{p}\left(\tau_{p}\right) \int_{t_{a}=0}^{\tau_{p}} f_{a}\left(t_{a}\right) d t_{a} d \tau_{p} \\
& =\int_{\tau_{p}=0}^{\infty} r_{p}\left(\tau_{p}\right) F_{a}\left(\tau_{p}\right) d \tau_{p} \\
& =\left(\frac{1}{2 \pi i}\right) \int_{\sigma-i \infty}^{\sigma+i \infty} \int_{\tau_{p}=0}^{\infty} r_{p}\left(\tau_{p}\right) e^{s \tau_{p}}\left[\frac{f_{a}^{*}(s)}{s}\right] d \tau_{p} d s \\
& =\left(\frac{1}{2 \pi i}\right) \int_{\sigma-i \infty}^{\sigma+i \infty} r_{p}^{*}(-s)\left[\frac{f_{a}^{*}(s)}{s}\right] d s \\
& =-\sum_{z \in \sigma_{p}} \operatorname{Res}_{s=z} r_{p}^{*}(-s)\left[\frac{f_{a}^{*}(s)}{s}\right]
\end{aligned}
$$

where $i=\sqrt{-1}, \sigma$ is a sufficiently small positive number, $\sigma_{p}$ is the set of poles of $r_{p}^{*}(-s)$ in the right half of the complex plane, and $\operatorname{Res}_{s=z}$ denotes the residue at the pole $s=z$. Alternatively, $\operatorname{Pr}\left[t_{a}<\tau_{p}\right]$ can be also derived as

$$
\begin{aligned}
\operatorname{Pr}\left[t_{a}<\tau_{p}\right] & =\int_{t_{a}=0}^{\infty} f_{a}\left(t_{a}\right) \int_{\tau_{p}=t_{a}}^{\infty} r_{p}\left(\tau_{p}\right) d \tau_{p} d t_{a} \\
& =\int_{t_{a}=0}^{\infty} f_{a}\left(t_{a}\right)\left[1-R_{p}(t)\right] d t_{a} \\
& =1-\left(\frac{1}{2 \pi i}\right) \int_{\sigma-i \infty}^{\sigma+i \infty} \int_{t_{a}=0}^{\infty} f_{a}\left(t_{a}\right) e^{s t_{a}}\left[\frac{r_{p}^{*}(s)}{s}\right] d t_{a} d s \\
& =1+\sum_{z \in \sigma_{a}} \operatorname{Res}_{s=z} f_{a}^{*}(-s)\left[\frac{r_{p}^{*}(s)}{s}\right]
\end{aligned}
$$

where $\sigma_{a}$ is the set of poles of $f_{a}^{*}(-s)$ in the right half of the complex plane. 
For demonstration purposes, we compute $\operatorname{Pr}\left[t_{a}<\tau_{p}\right]$ based on two cases of $t_{a}$ and $t_{p}$ distributions.

Case 1: $t_{a}$ is a Gamma random variable with shape parameter $k$ and rate parameter $\lambda$, and $t_{p}$ is an Erlang random variable with shape parameter $m$ and rate parameter $\gamma$. In this case, $k>0$ is a real number, and $m \geq 1$ is an integer.

Case 2: $t_{a}$ is an Erlang random variable with shape parameter $k$ and rate parameter $\lambda$, and $t_{p}$ is a Gamma random variable with shape parameter $m$ and rate parameter $\gamma$. In this case, $k \geq 1$ is an integer, and $m>0$ is a real number.

The Gamma distribution is considered because this distribution is widely used in telecom modeling [14]-[17]. We also select the Erlang distribution because this distribution can be easily extended into a hyper Erlang distribution, which has been proven to be a good approximation to many other distributions and measured data [13], [18]. The Laplace transforms for the $t_{a}$ and the $t_{p}$ are

$$
f_{a}^{*}(s)=\left(\frac{\lambda}{s+\lambda}\right)^{k} \quad f_{p}^{*}(s)=\left(\frac{\gamma}{s+\gamma}\right)^{m} .
$$

For Gamma $t_{a}$ and $t_{p}, k$ and $m>0$ are positive real numbers in (4). For Erlang $t_{a}$ and $t_{p}, k$ and $m$ are positive integer numbers. From the residual life theorem [10] and (4), $r_{p}^{*}(s)$ is expressed as

$r_{p}^{*}(s)$

$$
=\left(\frac{\gamma}{s m}\right)\left[1-f_{p}^{*}(s)\right]=\left(\frac{\gamma}{s m}\right)\left[1-\left(\frac{\gamma}{s+\gamma}\right)^{m}\right] .
$$

For Case 1, we substitute (4) and (5) into (2) to yield

$$
\begin{aligned}
\operatorname{Pr}\left[t_{a}<\tau_{p}\right]=\sum_{i=0}^{m-1} \sum_{j=0}^{i} & {\left[\frac{\gamma^{i-j}}{m \lambda^{i-j}(i-j) !}\right] } \\
& \times\left(\frac{\lambda}{\gamma+\lambda}\right)^{k+i-j} \prod_{l=1}^{i-j}(k+l-1) .
\end{aligned}
$$

Note that, in (6), when $i-j=0, \prod_{l=1}^{i-j}$ represents an empty product, and its value is 1 . From (1) and (6), $p$ is rewritten as

$$
\begin{aligned}
& p=\sum_{i=0}^{m-1} \sum_{j=0}^{i}\left[\frac{\mu \gamma^{i-j}}{(\gamma+m \mu) \lambda^{i-j}(i-j) !}\right] \\
& \times\left(\frac{\lambda}{\gamma+\lambda}\right)^{k+i-j} \prod_{l=1}^{i-j}(k+l-1) .
\end{aligned}
$$

On the other hand, for Case 2, from (4) and (5), (3) is rewritten as

$$
\begin{aligned}
\operatorname{Pr}\left[t_{a}<\tau_{p}\right]=1- & \sum_{i=0}^{k-1}\left\{\frac{\gamma}{\lambda m}-\sum_{j=0}^{i}\left(\frac{\lambda^{j-1}}{j ! m \gamma^{j-1}}\right)\right. \\
& \left.\times\left(\frac{\gamma}{\lambda+\gamma}\right)^{m+j} \prod_{l=1}^{j}(m+l-1)\right\} .
\end{aligned}
$$

From (1) and (8), $p$ is rewritten as

$$
\begin{aligned}
p=\left(\frac{m \mu}{\gamma+m \mu}\right)\{ & 1-\sum_{i=0}^{k-1}\left\{\frac{\gamma}{\lambda m}-\sum_{j=0}^{i}\left(\frac{\lambda^{j-1}}{j ! m \gamma^{j-1}}\right)\right. \\
& \left.\left.\times\left(\frac{\gamma}{\lambda+\gamma}\right)^{m+j} \prod_{l=1}^{j}(m+l-1)\right\}\right\} .
\end{aligned}
$$

Equations (7) and (9) are validated against the discrete-event simulation experiments, which shows that the discrepancies between the analytic and simulation results are within $0.5 \%$.

\section{NUMERICAL EXAMPLES}

Here, we study the call setup delays of DR and IR. Let $t_{f}$ be the time that the UE falls back from LTE to UMTS (i.e., Steps 1-10 in Fig. 2). Let $t_{d}$ be the UMTS outgoing call setup delay without the CSFB (i.e., Steps $11-15$ in Fig. 2). Then, the performance improvement $\alpha$ of the DR scheme over the IR scheme can be defined as

$$
\alpha=1-\frac{(1-p) E\left[t_{f}\right]+E\left[t_{d}\right]}{E\left[t_{f}\right]+E\left[t_{d}\right]}=\frac{p E\left[t_{f}\right]}{E\left[t_{f}\right]+E\left[t_{d}\right]} .
$$

In (10), $E\left[t_{f}\right]+E\left[t_{d}\right]$ is the expected total call setup delay for $\mathrm{IR}$, and $(1-p) E\left[t_{f}\right]+E\left[t_{d}\right]$ is the expected total call setup delay for DR. The larger the $\alpha$ value, the better the performance of DR over IR. From the call setup delay measurement of Qualcomm (see Section II), $E\left[t_{f}\right]=2.5 \mathrm{~s}$ for 3GPP R8, $E\left[t_{f}\right]=0.5 \mathrm{~s}$ for $3 \mathrm{GPP} \mathrm{R} 9$, and $E\left[t_{d}\right]=4 \mathrm{~s}$. From Huawei's measurements [6], $E\left[t_{f}\right]=9 \mathrm{~s}$ for $3 \mathrm{GPP} R 8, E\left[t_{f}\right]=3 \mathrm{~s}$ for $3 \mathrm{GPP}$ R9, and $E\left[t_{d}\right]=5 \mathrm{~s}$. We also measured the call setup delay at Broadband Mobile Laboratory of National Chiao Tung University [7], where $E\left[t_{f}\right]$ is more than $10 \mathrm{~s}$ and $E\left[t_{d}\right]=7 \mathrm{~s}$. Our measurement results are more consistent with Huawei's results than that of Qualcomm's results. In this paper, we use Huawei's results to compute $\alpha$ in (10).

We also note that, although an LTE data connection is "always on," the connection is in the idle mode (and is actually disconnected) if no data session is in progress. Because the expected session holding time is typically shorter than the expected intersession arrival time [19], [20], we assume that $0.01 E\left[t_{p}\right] \leq E\left[t_{s}\right] \leq 0.1 E\left[t_{p}\right]$. We consider the effects of $t_{s}$ (the session holding time), $t_{a}$ (the intercall arrival time), and $t_{p}$ (the intersession arrival time) on the probability $p$ that a voice call can be connected without the CSFB overhead. Note that the voice call holding time $t_{c}$ does not affect $p$ and is not considered. We also note that the $\alpha$ value is proportional to the $p$ value [see (10)], and the effects on $\alpha$ are similar to those on $p$. Finally, to simplify our discussion, $t_{s}$ and $t_{a}$ are normalized by $t_{p}$.

Effects of $E\left[t_{s}\right] / E\left[t_{p}\right]$ : Fig. 7(a) shows that $p$ decreases as $E\left[t_{s}\right] / E\left[t_{p}\right]$ increases. When $E\left[t_{s}\right] / E\left[t_{p}\right]$ increases, a call is more likely to be released in the $t_{s}$ interval. In this case, the UE will return to LTE immediately, and smaller $p$ is observed. The nontrivial observation is that $E\left[t_{s}\right] / E\left[t_{p}\right]$ has an insignificant impact on $p$ for all $E\left[t_{s}\right] / E\left[t_{p}\right]$ values under our study. The probability $p$ and the improvement 


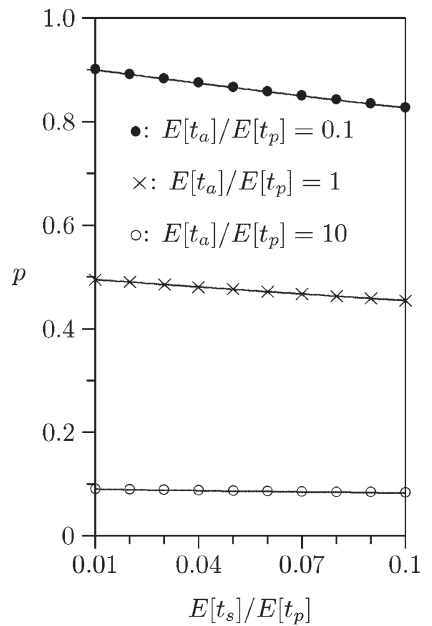

(a)

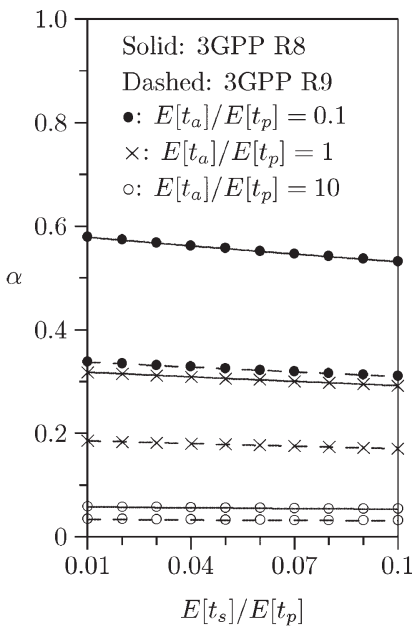

(b)
Fig. 7. Effects of $E\left[t_{s}\right] / E\left[t_{p}\right]$ and $E\left[t_{a}\right] / E\left[t_{p}\right]$ on $p$ and $\alpha\left(V_{a}=E\left[t_{a}\right]^{2}\right.$ and $V_{p}=E\left[t_{p}\right]^{2}$ ). (a) Effects on $p$. (b) Effects on $\alpha$.

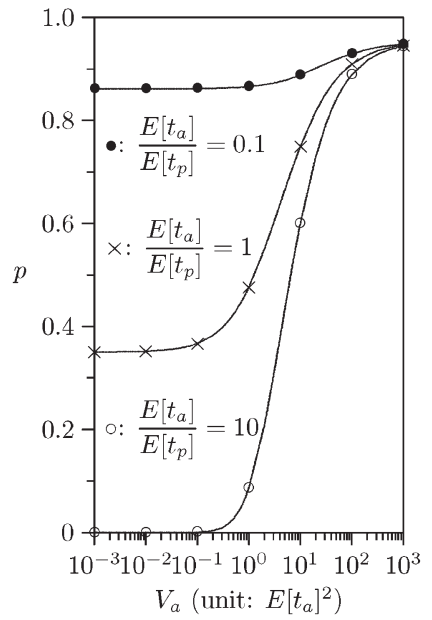

(a)

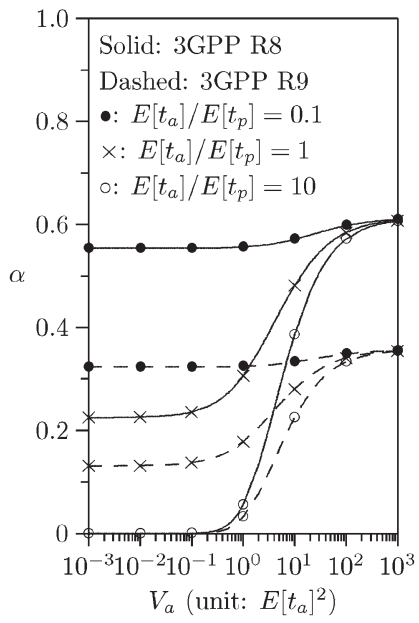

(b)
Fig. 8. Effects of $V_{a}$ and $E\left[t_{a}\right] / E\left[t_{p}\right]$ on $p$ and $\alpha\left(E\left[t_{s}\right] / E\left[t_{p}\right]=0.05\right.$ and $V_{p}=E\left[t_{p}\right]^{2}$ ). (a) Effects on $p$. (b) Effects on $\alpha$.

$\alpha$ decrease by $8 \%$ when $E\left[t_{s}\right]$ increases from $0.01 E\left[t_{p}\right]$ to $0.1 E\left[t_{p}\right]$. In other words, in this operational range, we can ignore the effect of $E\left[t_{s}\right] / E\left[t_{p}\right]$ and can focus more on other parameters.

Effects of $E\left[t_{a}\right] / E\left[t_{p}\right]$ : Figs. 7(a)-9(a) indicate that $p$ decreases as $E\left[t_{a}\right] / E\left[t_{p}\right]$ increases. When $E\left[t_{a}\right] / E\left[t_{p}\right]$ increases, the data session is more likely to arrive before the voice call arrives (i.e., the UE will return to LTE before the next call arrives). Thus, a smaller $p$ is observed. Figs. 8(a) and 9(a) shows that the effects of $E\left[t_{a}\right] / E\left[t_{p}\right]$ become insignificant when $V_{a}$ or $V_{p}$ is large, where large $p$ and $\alpha$ are always observed.

Effects of $V_{a}$ : Fig. 8(a) indicates that $p$ increases as $V_{a}$ increases. For a fixed $E\left[t_{a}\right]$ value, when $V_{a}$ increases, there are much more short $t_{a}$ intervals than long $t_{a}$ intervals. For short $t_{a}$, it is very likely that $t_{a}<\tau_{p}$ (i.e., larger $\operatorname{Pr}\left[t_{a}<\right.$ $\left.\tau_{p}\right]$ is observed). From (1) and (10), $p$ and $\alpha$ increase as $V_{a}$ increases.

Effects of $V_{p}$ : Fig. 9(a) shows that $p$ increases as $V_{p}$ increases. When the intersession arrival interval becomes more irreg-

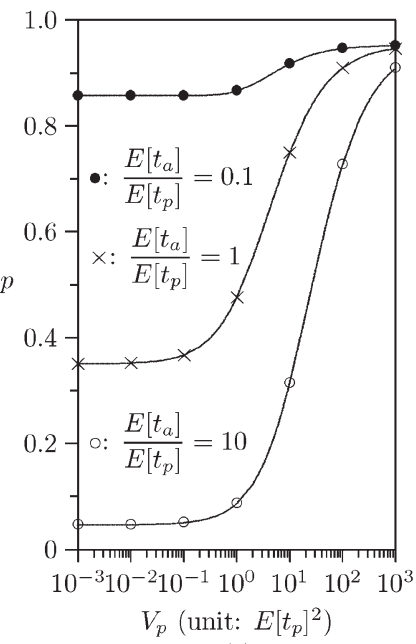

(a)

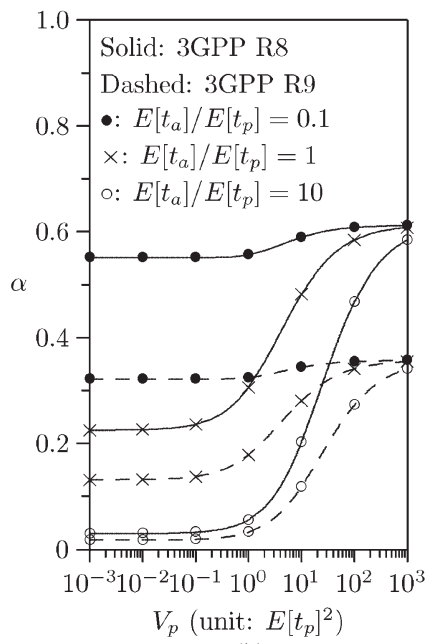

(b)
Fig. 9. Effects of $V_{p}$ and $E\left[t_{a}\right] / E\left[t_{p}\right]$ on $p$ and $\alpha\left(E\left[t_{s}\right] / E\left[t_{p}\right]=0.05\right.$ and $V_{a}=E\left[t_{a}\right]^{2}$ ). (a) Effects on $p$. (b) Effects on $\alpha$.

ular (i.e., $V_{p}$ increases), more long and short $t_{p}$ intervals are observed. Since the call release events are more likely to fall in long $t_{p}$ intervals and the next calls are likely to arrive before the next sessions arrive, larger $p$ and $\alpha$ are observed.

Based on (10) and Figs. 7(a)-9(a), Figs. 7(b)-9(b) plot the $\alpha$ curves against $E\left[t_{s}\right] / E\left[t_{p}\right], V_{a}$, and $V_{p}$. These figures show, that with probability $p$, the CSFB with DR can reduce outgoing call setup delay over the CSFB with IR by up to $60 \%$. We note that the DR scheme can also reduce the incoming call setup delay (i.e., the delay between when the network pages the UE and when the UE rings). The incoming call setup delay is typically shorter than the outgoing call setup delay. From (10), the DR scheme has even better $\alpha$ performance for the incoming calls than that for the outgoing calls. Since the called party of a voice call does not experience call setup delay, the improvement $\alpha$ is only meaningful from the network cost viewpoint.

\section{Conclusion}

This paper has proposed the DR scheme to avoid unnecessary CSFBs. An analytic model has been developed based on real LTE/UMTS network measurements to compare the DR scheme with the existing IR scheme. The performance is measured by the probability $p$ that, when a voice call arrives, the UE can be connected at UMTS without CSFB; therefore, nonnecessary switching between UMTS and LTE is avoided. In other words, when a voice call arrives, the UE does not need to switch from LTE to UMTS, and when the call is complete, the UE does not need to switch from UMTS to LTE. This paper have shown that the DR scheme can effectively improve the CSFB performance in the following conditions.

- The intercall arrival time $t_{a}$ is short (i.e., the voice calls arrive frequently).

- The variance of $t_{a}$ is large (i.e., the intercall arrival time is irregular).

- The variance of the intersession arrival time $t_{p}$ is large (i.e., the intersession arrival time is irregular). 
The last two items in our conclusions are not trivial, and are used as guidelines to further investigate the user behavior by a commercial mobile operator. For users with long intercall arrival time and regular call and data session arrivals, the CSFB with IR is exercised, whereas for the users with short intercall arrival time and irregular call and data session arrivals, the CSFB with DR is exercised.

As a final remark, the DR scheme can be practically implemented in NodeB with a minor modification in the RRC Connection Release message. Therefore, the DR scheme is an effective approach for reducing the CSFB costs. In the future, we will investigate the DR scheme based on the call and data traffic statistics collected from the commercial mobile telecom network. We will also consider other approaches to avoiding unnecessary CSFBs (e.g., a timer-based scheme that determines the optimal time interval for the UE to stay in UMTS based on different traffic rates). Moreover, because both CSFB and Enhanced Single Radio Voice Call Continuity [21], [22] are voice call solutions in LTE, we will compare the call performance between these two solutions.

\section{REFERENCES}

[1] "3rd generation partnership project; Technical specification group services and system aspects; General Packet Radio Service (GPRS) enhancements for Evolved Universal Terrestrial Radio Access Network (E-UTRAN) access (release 12)," Sophia-Antipolis, France, Tech. Spec. 3GPP TS 23.401 V12.2.0, 2013.

[2] "3rd generation partnership project; Technical specification group services and system aspects; General Packet Radio Service (GPRS); Service description; Stage 2 (release 12)," Sophia-Antipolis, France, Tech. Spec. 3GPP TS 23.060 V12.2.0, 2013.

[3] "3rd generation partnership project; Technical specification group core network; Digital cellular telecommunications system (Phase 2+); Basic call handling; Technical realization (release 1998)," Sophia-Antipolis, France, Tech. Spec. 3GPP TS 03.18 V7.5.0, 2003.

[4] "3rd generation partnership project; Technical specification group services and system aspects; Circuit Switched (CS) fallback in Evolved Packet System (EPS); Stage 2 (release 11)," Sophia-Antipolis, France, Tech. Spec. 3GPP TS 23.272 V12.0.0, 2013.

[5] Circuit-Switched Fallback: The First Phase of Voice Evolution for Mobile LTE Devices, Qualcomm Inc., San Diego, CA, USA, 2013.

[6] Circuit-Switched Fallback, Ultra-Flash CSFB, Huawei Technologies Co. Ltd., Shenzhen, China, 2013.

[7] Y.-B. Lin, P.-J. Lin, Y. C. Sung, Y.-K. Chen, W.-E. Chen, N. Alrajeh, B.-S. P. Lin, and C.-H. Gan, "Performance measurements of TD-LTE, WiMAX and 3G systems," IEEE Wireless Commun., vol. 20, no. 3, pp. 153-160, Jun. 2013.

[8] Y.-B. Lin and A.-C. Pang, Wireless and Mobile All-IP Networks. Hoboken, NJ, USA: Wiley, 2005.

[9] "3rd generation partnership project technical specification group radio access network; Radio Resource Control (RRC); Protocol specification (release 11)," Sophia-Antipolis, France, Tech. Spec. 3GPP TS 25.331 V11.7.0, 2013.

[10] L. Kleinrock, Queueing Systems, Vol. I: Theory. Hoboken, NJ, USA: Wiley, 1976.

[11] S. M. Ross, Stochastic Processes. Hoboken, NJ, USA: Wiley, 1996.

[12] W. R. LePage, Complex Variables and the Laplace Transform for Engineers. New York, NY, USA: Dover, 1980.

[13] Y. Fang and I. Chlamtac, "Teletraffic analysis and mobility modeling of PCS networks," IEEE Trans. Commun., vol. 47, no. 7, pp. 1062-1072, Jul. 1999.

[14] I. F. Akyildiz, J. S. M. Ho, and Y.-B. Lin, "Movement-based location update and selective paging for PCS networks," IEEE/ACM Trans. Netw., vol. 4, no. 4, pp. 629-638, Aug. 1996.

[15] D.-W. Huang, P. Lin, and C.-H. Gan, "Design and performance study for a mobility management mechanism (WMM) using location cache for wireless mesh network," IEEE Trans. Mobile Comput., vol. 7, no. 5, pp. 546-556, May 2008.
[16] C.-Y. Hong and A.-C. Pang, "3-approximation algorithm for joint routing and link scheduling in wireless relay networks," IEEE Trans. Wireless Commun., vol. 8, no. 2, pp. 856-861, Feb. 2009.

[17] R.-H. Liou, Y.-B. Lin, and S.-C. Tsai, "An investigation on LTE mobility management," IEEE Trans. Mobile Comput., vol. 12, no. 2, pp. 166-176, Jan. 2013.

[18] F. Kelly, Reversibility and Stochastic Networks. Hoboken, NJ, USA: Wiley, 1979.

[19] J. Färber, S. Bodamer, and J. Charzinski, "Measurement and modelling of Internet traffic at access networks," in Proc. EUNICE, Munich, Germany, 1998, pp. 196-203.

[20] J. Ferreira and F. J. Velez, "Enhanced UMTS services and applications characterization," Telektronikk, vol. 101, no. 1, pp. 113-131, Mar. 2005.

[21] "3rd generation partnership project; Technical specification group services and system aspects; IP Multimedia (IM) Core Network (CN) subsystem IP Multimedia Subsystem (IMS) service continuity; Stage 3,' Sophia-Antipolis, France, Tech. Spec. 3GPP TS 24.237 V12.2.0, 2013.

[22] Y.-B. Lin, R.-H. Liou, Y. C. Sung, and P.-C. Cheng, "Performance evaluation of LTE eSRVCC with limited access transfers," IEEE Trans. Wireless Commun., vol. 13, no. 5, pp. 2402-2411, May 2014.

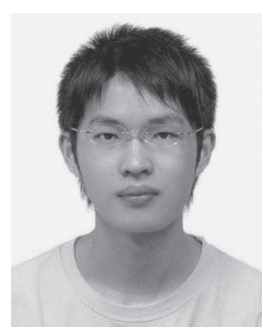

Ren-Huang Liou received the B.S. and M.S. degrees in computer science from National Chiao Tung University, Hsinchu, Taiwan, in 2007 and 2009, respectively, where he is currently working toward the Ph.D. degree.

His current research interests include Voice over Internet Protocol, mobile computing, and performance modeling.

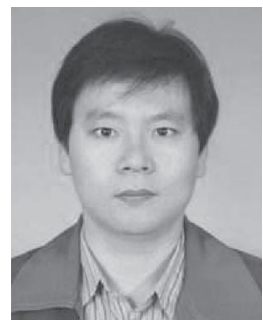

Yi-Bing Lin (M'96-SM'96-F'03) received the B.S. degree in electrical engineering from National Cheng Kung University (NCKU), Tainan, Taiwan, in 1983 and the Ph.D. degree in computer science from University of Washington, Seattle, WA, USA, in 1990.

$\mathrm{He}$ is currently the Senior Vice President and a Life Chair Professor with the Department of Computer Science, National Chiao Tung University, Hsinchu, Taiwan. He is also with the Institute of Information Science and the Research Center for Information Technology Innovation, Academia Sinica, Taipei, Taiwan. He is a member of the board of directors of Chunghwa Telecom. $\mathrm{He}$ is the author of Wireless and Mobile Network Architecture (Wiley, 2001), Wireless and Mobile All-IP Networks (Wiley, 2005), and Charging for Mobile All-IP Telecommunications (Wiley, 2008).

Dr. Lin is a Fellow of the Association for Computing Machinery, the American Association for the Advancement of Science, and the Institution of Engineering and Technology. He has received numerous research awards, including the 2005 National Science Council Distinguished Researcher and the 2006 Academic Award of Ministry of Education.

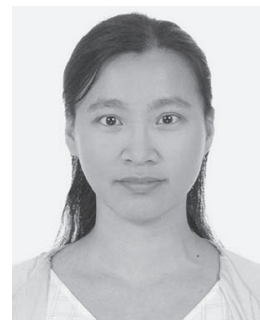

Yingrong Coral Sung received the B.S. and M.S degrees in computer science and information engineering and the Ph.D. degree from National Chiao Tung University (NCTU), Hsinchu, Taiwan, in 2002, 2003, and 2010, respectively.

She is currently an Assistant Research Fellow with the Center for Information and Communications Technology, NCTU. Her current research interests include the design and analysis of personal communications service networks, network security, and performance modeling. 


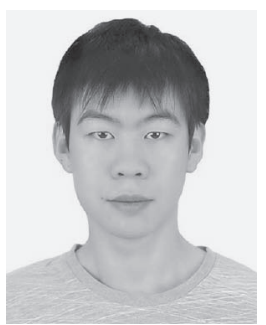

Pang-Cheng Liu received the B.S. degree in computer science from National Chiao Tung University, Hsinchu, Taiwan, in 2012, where he is currently working toward the M.S. degree with the Department of Computer Science.

His current research interests includes mobile network standard (Universal Mobile Telecommunication System/Long Term Evolution) and softwaredefined networks.

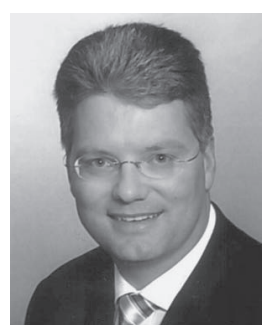

Christian Wietfeld (M'05-SM'12) received the Dipl.-Ing. and Dr.-Ing. degrees from RWTH Aachen University, Aachen, Germany.

He is currently a Full Professor of communication networks and the Head of the Communication Networks Institute, Technische Universität Dortmund, Dortmund, Germany. For more than 20 years, he has been a coordinator of and a contributor to large-scale research projects on Internet-based mobile communication systems in academia and industry (Siemens $\mathrm{AG})$. He is the author of over 175 peer-reviewed

papers and patents.

Dr. Wietfeld is the Co-Chair of the Committee on "Communication Networks and Systems" of the Information Technology Society of the Association for Electrical, Electronic and Information Technologies (VDE), the IEEE German sister organization. He is a Co-Founder of the IEEE Global Communications Conference Workshop on Wireless Networking for Unmanned Autonomous Vehicles. He is a member of the editorial board of the Transactions on Emerging Telecommunication Technologies (Wiley). He received an ITU-T Outstanding Contribution Award for his work on the standardization of next-generation mobile network architectures. 